\title{
SEARCHING FOR COLOR OCTET TAU LEPTONS AT HIGH ENERGY LEPTON- LEPTON AND HADRON-HADRON COLLIDERS
}

\author{
Metin $\operatorname{KANTAR}^{1}$ (D) \\ ${ }^{1}$ Department of Physics /Faculty of Sciences, Muğla University/Institution, 48000, Muğla, Turkey \\ metin@mu.edu.tr
}

Received: 13.06.2017, Accepted: 31.10.2017

*Corresponding author

doi: $10.22531 /$ muglajsci.321234

\section{Abstract}

In this study we search for pair production of color octet tau leptons and their decay modes at high energy $\tau^{+} \tau^{-}$colliders such as TESLA$\tau \times C L I C$ (TESLA- $\tau$, the tau version of TESLA) with the energy of 5, 10 and $15 \mathrm{TeV}$ and pp colliders such as LHC (Large Hadron Collider) with the energy of $14 \mathrm{TeV}, \mathrm{VLHC}$ (Very Large Hadron Collider) with the energy of $28 \mathrm{TeV}$ and SSC (Superconducting Super Collider) with the energy of $40 \mathrm{TeV}$. The total cross sections of color octet tau leptons for the three different machines group (at both $\tau^{+} \tau^{-}$and $p p$ colliders) are calculated and plotted versus its mass. The signal events for color octet tau leptons are analyzed by means of the cross section. The observation conditions of color octet tau leptons are performed by setting the discovery limits with the statistical significant event numbers.

Keywords: Cross Section, Luminosity, Number of Events, Standard Model

\section{YÜKSEK ENERJİLİ LEPTON-LEPTON VE HADRON-HADRON ÇARPIŞTIRICILARDA RENK SEKIZLİSI TAU LEPTONLARIN ARANMASI}

\section{Özet}

Bu çalışmada renk sekizlisi tau leptonların, sözgelimi 5, 10 ve 15 TeV enerjili, TESLA- $\tau \times C L I C$ (TESLA- $\tau$, TESLA'nın tau versiyonu) gibi yüksek enerjili $\tau^{+} \tau^{-}$çarpıştırıcılarında ve 14 TeV enerjili LHC (Büyük Hadron Çarpıştırıcısı), 28 TeV enerjili VLHC (Çok Büyük Hadron Çarpış̧ırıcısı) ve $40 \mathrm{TeV}$ enerjili SSC (Süperiletken Süper Çarpış̧ırıcı) gibi yüksek enerjili pp çarpıştırıcılarında, çift üretimlerini ve bozunum kanallarını araştırmaktayız. Üç farklı makine grubu için (hem $\tau^{+} \tau^{-}$hem de pp çarpıştırıcıları) renk sekizlisi tau leptonların toplam tesir kesiti hesaplanmakta ve kütlesine göre grafiği çizilmektedir. Bu parçacıklar için sinyal olayları tesir kesitine bağlı olarak analiz edilmektedir. Ayrıca bu parçacıkların gözlenebilme koşulları istatistiksel anlamlı olay sayılarıyla birlikte, keşfedilme sınırları ayarlanarak çalışılmıștır.

Anahtar Kelimeler: Standart Model, Kompozitlik, Işınlılık, Olay Sayısı, Tesir Kesiti

\section{Introduction}

The proliferation of the numbers of leptons and quarks has increased the speculations that they could be composite structures, i.e. bound states of more fundamental constituents often called "preons". On the other hand, the disability of the Standard Model (SM) to explain the most important fundamental problems such as lepton-quark-symmetry, family replication, mass hierarchy problem, divergences at Higgs mass, charge quantization, etc. forces the physicists to go beyond it. At this point, some approximations beyond SM such as compositeness, supersymmetry (SUSY), etc. might be the most promising candidates to search new physics [1-6]. At the future colliders with high center of mass energy and luminosity we may have large physics potential in order to explore the $\mathrm{TeV}$ or multi-TeV scale physics. So far, no obviously correct or compelling model has yet emerged. It is hoped that at the LHC the particles predicted by some composite models will be discovered.

In this study, we aim to give a realistic composite model for leptons and quarks which contains scalar and fermionic preons. In order to test our model, we suggest experimental researches on color octet tau lepton productions. Color octet tau leptons will be pairly produced in $\boldsymbol{\tau}^{+} \boldsymbol{\tau}^{-}$and $\boldsymbol{p} \boldsymbol{p}$ collisions. If we may observe these particles at the future high energy collider experiments, then it might be a signature of the compositeness of leptons and quarks. At high energy $\boldsymbol{\tau}^{+} \boldsymbol{\tau}^{-}$and pp colliders, we hope that there will be so much physics potential to search a new scale physics, or so called $\mathrm{TeV}$ scale physics and a new type fundamental interactions. In the future colliders it may be possible to search for a lot of exotic particles.

At present there are many ideas on compositeness [7-11]. These ideas start from the compositeness of Higgs particle to all particles of the Standard Model. In these models, all the SM particles are consist of more fundamental constituents so called preons. Unfortunately, there is no experimental evidence on the existence of lepton and quark compositness at moment. To make a model we have some assumptions to restrict the number of parameters. There are two assumptions to our preon model,

i) There is no parastatistics,

ii) Preons are colored object. 
According to the first assumption, the SM fermions should contain odd number of fermionic preons, which lead to fermion scalar models $[9,12-17]$ or three fermion models $[8,15]$. The second assumption means that all preons are color triplets.

Leptons: In the framework of fermion scalar models, leptons would be a bound state of one fermionic preon and one scalar anti-preon,

$$
l=(F \bar{S})=1 \oplus 8
$$

Then each SM lepton has one color octet partner. In a three fermion, the color decomposition

$$
l=(F F F)=1 \oplus 8 \oplus 8 \oplus 10
$$

Predicts the existence of two color octet and one color decouplet partners.

Quarks: In fermion scalar models, anti-quarks are made of one fermionic and one scalar preons which means that each SM anti-quark has one color sextet partner,

$$
q=(F S)=\overline{3} \oplus 6
$$

In three fermion models,

$$
\begin{array}{lr}
q=(F \bar{F} F)=3 \oplus \overline{3} \oplus 6 \oplus 15 \\
\boldsymbol{v}_{\boldsymbol{e}}=\left(\boldsymbol{F}_{\mathbf{1}} \overline{\boldsymbol{S}}_{\mathbf{1}}\right), & \boldsymbol{e}=\left(\boldsymbol{F}_{\mathbf{2}} \overline{\boldsymbol{S}}_{\mathbf{1}}\right) \\
\overline{\boldsymbol{d}}=\left(\boldsymbol{F}_{\mathbf{1}} \boldsymbol{S}_{\mathbf{2}}\right), & \overline{\boldsymbol{u}}=\left(\boldsymbol{F}_{\mathbf{2}} \boldsymbol{S}_{\mathbf{2}}\right)
\end{array}
$$

\section{Production of Color Octet Tau Leptons}

\section{$2.1 \tau^{+} \tau^{-}$Colliders}

Composite models of leptons and quarks may have a number of new exotic particles such as leptogluons, leptoquarks, excited leptons, etc., The interaction Lagrangian between a lepton, a gluon and a leptogluon may be [18]

$$
\mathcal{L}=\frac{g_{s}}{2 \Lambda} \sum\left[\bar{l}_{8}^{\alpha} \sigma^{\mu \nu} F_{\mu \nu}^{\alpha}\left(\eta_{L} l_{L}+\eta_{R} l_{R}\right)+h . c\right]
$$

According to Eq. (6) it is possible to produce color octet tau leptons pairly in the $\mathrm{t}$-channel at $\tau^{+} \tau^{-}$colliders as

$$
\tau^{+} \tau^{-} \rightarrow \tau_{8}^{+} \tau_{8}^{-} \rightarrow 2 \mathrm{l}+2 \mathrm{jet}
$$

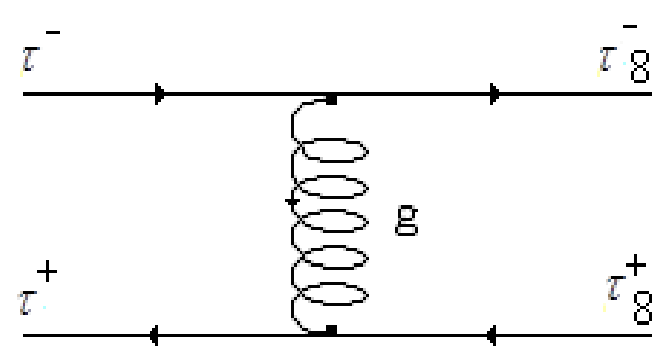

Figure 1. Feynman diagram for the pair production of color octet tau leptons coming from the t-channel at $\tau^{+} \tau^{-}$colliders.

In addition to this channel, an extra contribution coming from the contact interactions will be as [19]

$$
\mathcal{L}_{\text {int }} \approx \frac{g^{2}}{2 \Lambda^{2}} \tau_{8}^{+} \tau_{8}^{-} \tau^{+} \tau^{-}
$$

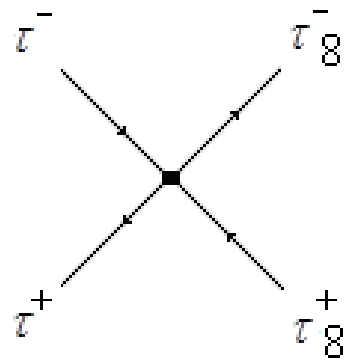

Figure 2. Feynman diagram for the pair production of color octet tau leptons coming from the contact interactions at $\boldsymbol{\tau}^{+} \boldsymbol{\tau}^{-}$ colliders.

From the diagrams seen in Fig. 1 and Fig. 2 we can easily calculate the differential cross section for color octet tau lepton production as

$$
\frac{d \widehat{\sigma}}{d \hat{t}}=\frac{\pi \alpha_{s}^{2}}{16 \Lambda^{4} \hat{s}^{2}}\left\{\hat{s}\left(\hat{s}-2 m^{2}\right)+16 k^{2}\left(\hat{t}-m^{2}\right)^{2}\right\}
$$

Where $k$ is the new interaction parameter seen in the contact interactions, $g^{2}=k g_{s}^{2}$, and $g$ is the new coupling constant for the four vertex interaction, $\Lambda$ is compositeness scale. If $k=1$, namely taking $\Lambda=m$, the contact interactions will be order of QCD coupling constant $\alpha_{s}$, but we don't know what will be. The total cross section for color octet tau lepton production is plotted versus its mass in Fig. 3. For $k=1$. As seen in Fig. 3, color octet tau lepton cross section is potentially large, then we might expect the number of events so large in $\tau^{+} \tau^{-}$collisions. On the other hand, there will be very clean signals without background events at $\tau^{+} \tau^{-}$colliders. Because we expect that color octet tau leptons will decay into two taus and two jets. For the various values of $k$, for example, $0.1,0.01$ and 0.001 , we obtained that the total cross section curves behave same compared to Fig. 3.

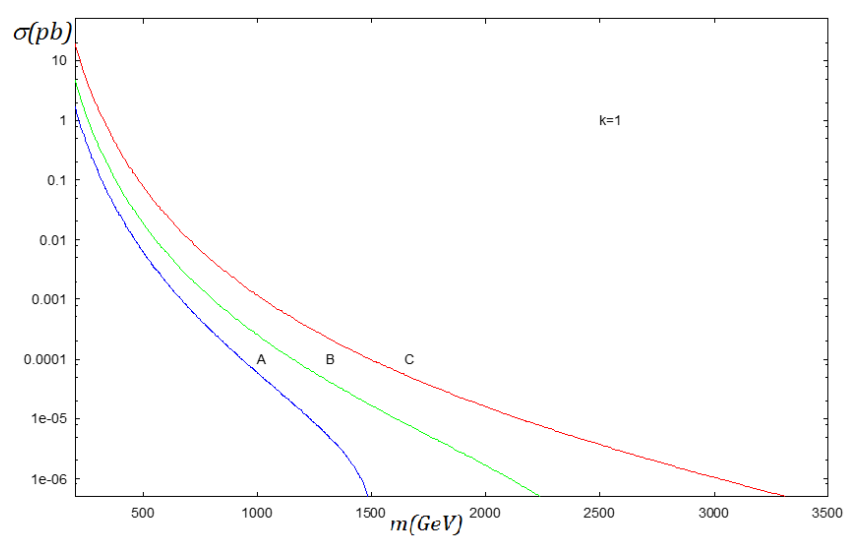

Figure 3. Total cross section for color octet tau leptons versus its mass $m$ setting to three different machines taking $k=1$, curve A with $\sqrt{s}=5 \mathrm{TeV}$, curve B with $\sqrt{s}=10 \mathrm{TeV}$ and curve C with $\sqrt{s}=15 \mathrm{TeV}$ at $\tau^{+} \tau^{-}$colliders

and its Feynman diagram as 


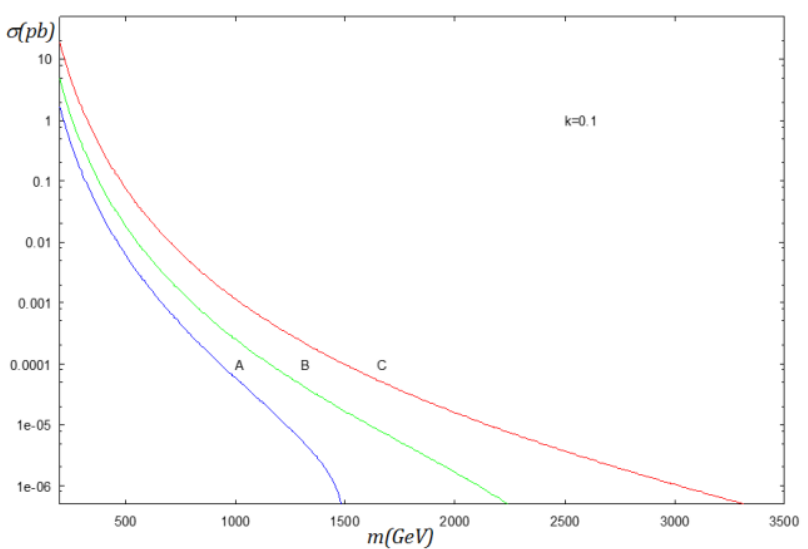

Figure 4. Total cross section for color octet tau leptons versus its mass $m$ setting to three different machines taking $k=0.1$, curve A with $\sqrt{s}=5 \mathrm{TeV}$, curve B with $\sqrt{s}=10 \mathrm{TeV}$ and curve C with $\sqrt{s}=15 \mathrm{TeV}$ at $\tau^{+} \tau^{-}$colliders.

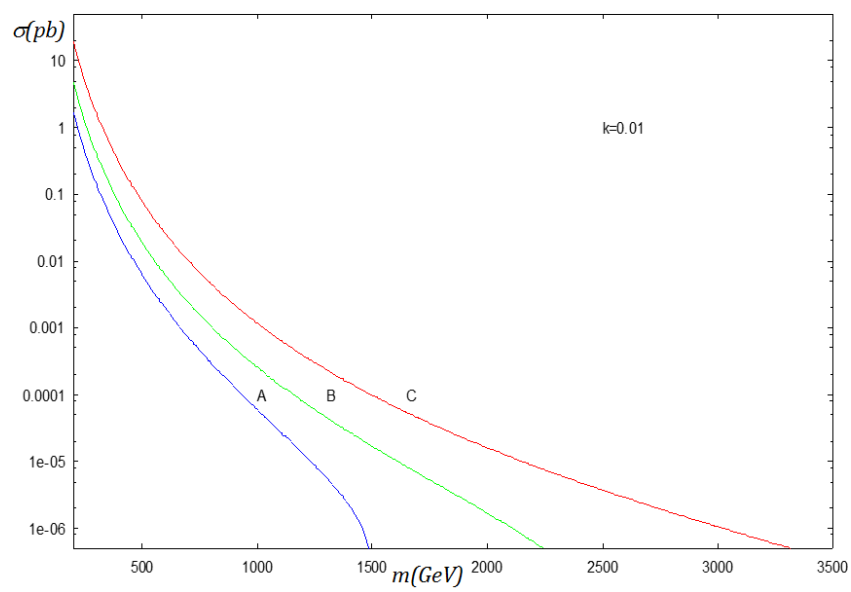

Figure 5. Total cross section for color octet tau leptons versus its mass $m$ setting to three different machines taking $k=0.01$, curve A with $\sqrt{s}=5 \mathrm{TeV}$, curve B with $\sqrt{s}=10 \mathrm{TeV}$ and curve C with $\sqrt{s}=15 \mathrm{TeV}$ at $\tau^{+} \tau^{-}$colliders.

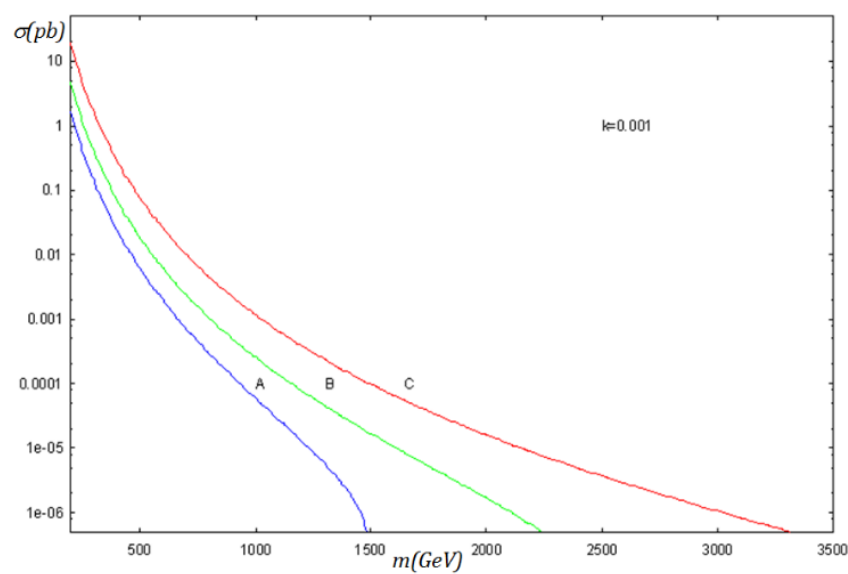

Figure 6. Total cross section for color octet tau leptons versus its mass $m$ setting to three different machines taking $k=0.001$, curve A with $\sqrt{s}=5 \mathrm{TeV}$, curve B with $\sqrt{s}=10 \mathrm{TeV}$ and curve C with $\sqrt{s}=15 \mathrm{TeV}$ at $\tau^{+} \tau^{-}$colliders.

\section{2 pp Colliders}

At LHC (or VLHC, upraged version of LHC) and SSC, leptogluons will be pairly produced in the s-, $\mathrm{t}$ - and $\mathrm{u}$ - channels as $\mathrm{gg} \rightarrow l_{8} \bar{l}_{8} \rightarrow 2 l+2 j e t, \quad q \bar{q} \rightarrow l_{8} \bar{l}_{8} \rightarrow 2 l+2 j e t$

and their Feynman diagrams are given as

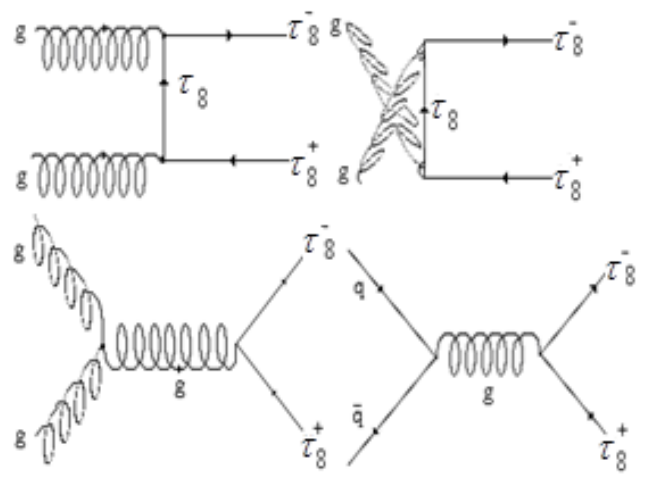

Figure 7. Feynman diagrams for the pair production of color octet tau leptons at pp colliders.

From these diagrams we can easily obtain the differential cross section for color octet tau lepton production coming from gluon-gluon fusion as

$$
\begin{gathered}
\frac{d \hat{\sigma}}{d \hat{t}}=d_{R} C(R) \frac{\pi \alpha_{s}^{2}}{32 \hat{s}^{2}}\left\{\frac{6}{\hat{s}^{2}}\left(\hat{t}-m^{2}\right)\left(\hat{u}-m^{2}\right)\right. \\
+C(R) \frac{\left(\hat{t}-m^{2}\right)\left(\hat{u}-m^{2}\right)-2 m^{2}\left(\hat{t}+m^{2}\right)}{\left(\hat{t}-m^{2}\right)^{2}} \\
+\frac{3\left(\hat{t}-m^{2}\right)\left(\hat{u}-m^{2}\right)-2 m^{2}\left(\hat{s}-4 m^{2}\right)}{\hat{s}\left(\hat{t}-m^{2}\right)} \\
+C(R) \frac{\left(\hat{u}-m^{2}\right)\left(\hat{t}-m^{2}\right)-2 m^{2}\left(\hat{u}+m^{2}\right)}{\left(\hat{u}-m^{2}\right)^{2}} \\
+\frac{3\left(\hat{u}-m^{2}\right)\left(\hat{t}-m^{2}\right)-2 m^{2}\left(\hat{s}-4 m^{2}\right)}{\hat{s}\left(\hat{u}-m^{2}\right)} \\
\left.-(3-2 C(R)) \frac{m^{2}\left(\hat{s}-4 m^{2}\right)}{\left(\hat{t}-m^{2}\right)\left(\hat{u}-m^{2}\right)}\right\}
\end{gathered}
$$

and quark-antiquark annihilation as

$$
\frac{d \hat{\sigma}}{d \hat{t}}=d_{R} C(R) \frac{\pi \alpha_{s}^{2}}{9 \hat{s}^{2}}\left\{\frac{\left(\hat{t}-m^{2}\right)^{2}+\left(\hat{u}-m^{2}\right)^{2}+2 m^{2} \hat{s}}{\hat{s}^{2}}\right\}
$$

where $d_{R}$ is the dimension of the representation and $C(R)$ is the color factor of color octet tau leptons. In our calculations we have taken $d_{R}=8$ and $C(R)=\frac{4}{3}$ for gg fusion, $d_{R}=8$ and $C(R)=3$ for $q \bar{q}$ fusion. According to Eq. (11) and Eq. (12) after integrating over $\hat{t}$ we can calculate the total cross section for color octet tau lepton production by using the parton distribution functions given in Ref. [20] at $p p$ colliders. The total cross section for color octet tau lepton production are plotted versus its mass in Fig. 8 for $g g$ fusion Fig. 9 for $q \bar{q}$ annihilation. 


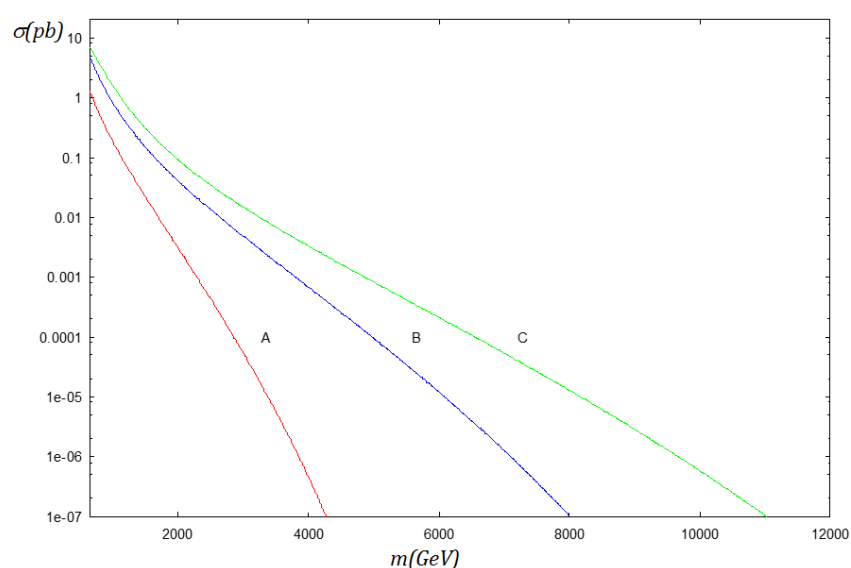

Figure 8. Total cross section for color octet tau leptons coming from the $g g$ fusion versus its mass $m$ setting to three different machines, curve A with $\sqrt{s}=14 \mathrm{TeV}$, curve B with $\sqrt{s}=28 \mathrm{TeV}$ and curve $\mathrm{C}$ with $\sqrt{s}=40 \mathrm{TeV}$ at $p p$ colliders.

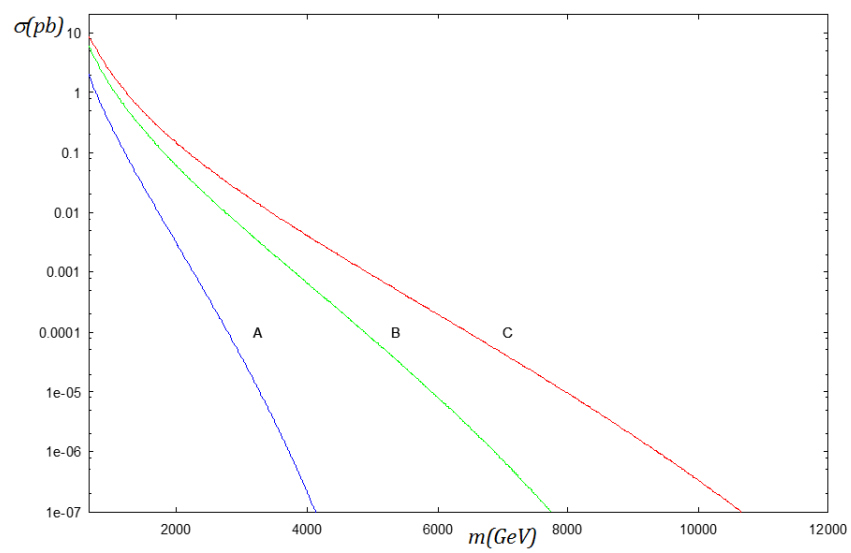

Figure 9. Total cross section for color octet tau leptons coming from the $q \bar{q}$ annihilation versus its mass $m$ setting to three different machines, curve A with $\sqrt{s}=$ $14 \mathrm{TeV}$, curve B with $\sqrt{s}=28 \mathrm{TeV}$ and curve C with $\sqrt{s}=$ $40 \mathrm{TeV}$ at $p p$ colliders.

\section{Conclusion}

In this work we assumed that leptogluons strongly interact with leptons by exchanging gluon in the t-channel according to effective Lagrangian (6). According to our calculations color octet tau leptons can be explored up to the masses of $0.98 \mathrm{TeV}$ for $\sqrt{s}=5 \mathrm{TeV}, 1.21 \mathrm{TeV}$ for $\sqrt{s}=10 \mathrm{TeV}$ and 1.52 $\mathrm{TeV}$ for $\sqrt{s}=15 \mathrm{TeV}$ in $\tau^{+} \tau^{-}$collisions, 2.72 (2.67) $\mathrm{TeV}$ for $\sqrt{s}=14 \mathrm{TeV}, 4.68$ (4.62) $\mathrm{TeV}$ for $\sqrt{s}=28 \mathrm{TeV}$ and 6.34 (6.20) $\mathrm{TeV}$ for $\sqrt{s}=40 \mathrm{TeV}$ in pp collisions. The values in the parenthesis correspond the mass limits coming from the subprocesses $q \bar{q}$. The existence of three types of leptogluons "colored leptons" is an essential feature of a large class of composite models of leptons and quarks. If they exist, both charged and neutral "colored leptons" are produced at appreciable rates and have extremely interesting experimental signatures.

\section{References}

[1]. Llewellyn Smith, C. H., "Supersymmetry and Its Experimental Consequences" Phys. Rep. 105, 5370,1984
[2]. Nilles, H. P., "Supersymmetry, Supergravity and Particle Physics", Phys. Rep. 110, 1-162, 1984,

[3]. Haber, H. E., Kane, G. L., "The Search for Supersymmetry: Probing Physics Beyond The Standard Model“, Phys. Rep. 117, 75-263, 1984,

[4]. Terazawa, H., "Subquark Model of Leptons and Quarks" Phys. Rev. 22, 184, 1980,

[5]. Harari, H., "Composite Model for Quarks and Leptons” Phys. Rep. 104, 159, 1984,

[6]. D’Souza I. A., and Kalman, C. S., "Preons: Models of Leptons, Quarks and Gauge Bosons as Composite Objects" World Scientific Publishing, 1992, 1-108,

[7]. Harari, H., "A Schematic Model of Quarks and Leptons", Phys. Lett. 86B, 83, 1979,

[8]. Harari, H. and Seiberg, N., "A Dynamical Theory for The Rishon Model”, Phys. Lett 98B, 269-273, 1981,

[9]. Shupe, M. A., "A Composite Model of Leptons and Quarks” Phys. Lett. 86B, 87-92, 1979,

[10]. Barbieri, R., Mohapatra, R. N. and Masiero, A., "Compositeness and A Left-Right Symmetric Electroweak Model Without Broken Gauge Interactions" Phys. Lett. 105B, 369-374, 1981,

[11]. Greenberg, 0. W. and Sucher, J., "A Quantum Structure Dynamic Model of Quarks, Leptons, Weak Vector Bosons and Higgs Bosons", Phys. Lett. 99B, 339-343, 1981,

[12]. Matumoto, K., "On A Composite Model for Hadronic Constituents", Prog. Theor. Phys. 52, 1973, 1974,

[13]. Greenberg, O. W. and Nelson, C. A., "Composite Model of Leptons" Phys. Rev. D 10, 2567, 1974,

[14]. Greenberg, 0. W., "Narrow Resonances Above 3 $\mathrm{GeV}$ and Separate Localization of Ordinary and Color SU(3)", Phys. Rev. Lett. 35, 1120, 1974,

[15]. Fritzsch, H. and Mandelbaum, G., "Weak Interactions as Manifestations of the Substructure of Leptons and Quarks", Phys. Lett. 102B, 319-322, 1981,

[16]. Sultanov, S., "Physics at Gamma-Proton Colliders of TeV Energies", in Proc of X Seminar Problems on High Energy Physics and Field Theory, Moskow, Nauka, 455, 1988,

[17]. Sultanov, S., "Leptonic Photon and Relic Neutinos", IHEP Preprint 87, Serpukhov, 87-119, 1987,

[18]. Hagiwara, K., Komamiya, S. and Zeppenfeld, D., "Excited Lepton Production at LEP and HERA", Z. Phys. C 29, 115, 1985,

[19]. Harari, H., “Colored Leptons", Phys. Lett. 156B, 250-254, 1985,

[20]. Martin, A. D., Stirling, W. J. and Roberts, R. G., "Pinning Down the Glue in The Proton", Phys. Lett. 354B, 155-162, 1995. 